\title{
Palisade technique as an effective endoscopic submucosal dissection tool for large colorectal tumors
}

\section{다 (1) $\odot$}

Authors

Yuichiro Suzuki ${ }^{1}$, Ken Ohata ${ }^{1}$, Eiji Sakai ${ }^{1}$, Ryoju Negishi ${ }^{1}$, Maiko Takita ${ }^{1}$, Yohei Minato ${ }^{1}$, Takeshi Muramoto ${ }^{1}$, Hideyuki Chiba $^{2}$, Yosuke Tsuji ${ }^{3}$, Nobuyuki Matsuhashi ${ }^{4}$

Institutions

1 Department of Gastrointestinal Endoscopy, NTT Medical Center, Tokyo, Japan

2 Department of Gastroenterology, Omori Red Cross hospital, Tokyo, Japan

3 Department of Gastroenterology, Graduate School of Medicine, The University of Tokyo, Tokyo, Japan

4 Department of Gastroenterology, NTT Medical Center Tokyo, Tokyo, Japan

submitted 3.6 .2020

accepted after revision $\quad 2.10 .2020$

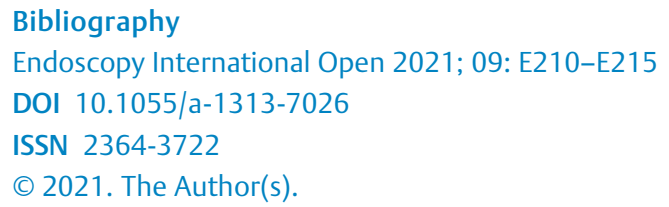
Commons Attribution-NonDerivative-NonCommercial License, permitting copying and reproduction so long as the original work is given appropriate credit. Contents may not be used for commecial purposes, or adapted, remixed, transformed or built upon. (https://creativecommons.org/licenses/by-nc-nd/4.0/)

Georg Thieme Verlag KG, Rüdigerstraße 14,

70469 Stuttgart, Germany

\section{Corresponding author}

Ken Ohata, MD, PhD, Division for Gastrointestinal Endoscopy, NTT Medical Center, 5-9-22 Higashi Gotanda, Shinagawa-ku, Tokyo, 141-0022, Japan

Fax: +81-30-3448-6111

ken.ohata1974@gmail.com

\section{ABSTRACT}

Background and study aims Endoscopic submucosal dissection (ESD) has become the standard treatment for colorectal ESD, but large colorectal tumors remain difficult to remove. We developed a new method, called the palisade technique, by modifying the multiple tunneling technique. In this method, a palisade of submucosal tissue is left beneath the tumor to anchor a dissected specimen, maintaining effective submucosal traction.

Patients and methods The study included 11 patients with large colorectal tumors that were over half the circumference of the colorectal lumen which were treated using the palisade technique from August 2017 to October 2019. Overall resection outcomes were assessed.

Results All 11 lesions were removed en bloc. The R0 resection rate was $45.6 \%$ because of marginal burning of the specimen, but no local recurrence was found after a median observation period of 31 months. The median submucosal dissection time (SDT) and submucosal dissection speed (SDS) were 170 minutes and $23.1 \mathrm{~mm}^{2} / \mathrm{min}$, respectively. One case of post-ESD hemorrhage was successfully managed endoscopically, and two cases of post-colorectal ESD coagulation syndrome were managed conservatively. Conclusion The palisade technique can be an effective and safe technique for treating large colorectal tumors that extend over half the luminal circumference.

\section{Introduction}

Endoscopic submucosal dissection (ESD) has become a popular method for treating early colorectal tumors, and its indication is broadening to target larger lesions. There has been a report of increased rates of adverse events (AEs) for lesions larger than $50 \mathrm{~mm}$, with size reported as an independent risk factor for post-ESD AEs [1]. In addition, expected fatigue from the long procedural time could deter endoscopists from performing ESD on larger lesions. Therefore, it is beneficial for both physicians and patients if a technique can be developed to effectively and safely dissect large tumors.

A previously reported technique, called the multiple tunneling technique, involves creation of multiple straight tunnels beneath the lesion [2]. The technique involves creating multiple straight submucosal tunnels from the anal to the oral side, purposely leaving multiple sheets of submucosal tissues between the tunnels, resembling a palisade. This palisade of submucosal tissues acts as an anchor to prevent the tumor mass from collapsing on the contralateral wall of the colon, which provides 
good traction throughout dissection. We believe that this property is the key to this technique and propose renaming the palisade technique as an improved descriptor.

We conducted a case series to investigate outcomes of colorectal ESD for large colorectal tumors using this technique to measure important resection outcomes, including R0 and enbloc resection rates, speed of dissection, and AEs.

\section{Patients and methods}

\section{Patients}

From August 2017 to October 2019, 837 colorectal ESDs were performed at NTT Medical Center Tokyo, Tokyo, Japan, a tertiary endoscopic center. Of these, 24 lesions were treated using the palisade technique. Thirteen lesions extended less than half of the luminal circumference and were excluded. The remaining 11 lesions that were over half the luminal circumference were retrospectively analyzed. Clinical data, including patient demographic details, lesion characteristics, and therapeutic outcomes were collected from our database.

This study was approved by the Ethics Committee of NTT Medical Center Tokyo (ID 19-286) and was registered with the University Hospital Medical Information Network (UMIN) Clinical Trials: UMIN 000038101. All ESDs were planned according to Japanese guidelines for ESD and endoscopic mucosal resection of colorectal cancer [3]. Antithrombotic and anticoagulant agents were stopped before the procedure in accordance with current guidelines [4].

\section{palisade technique}

All patients were sedated with intravenous flunitrazepam (conscious sedation). ESD was performed using a single-channel endoscope with water jet (PCF-H290AZI; Olympus Medical Systems, Tokyo, Japan). A transparent hood was used in all cases (Elastic touch, L, 16675; TOP co, Tokyo, Japan). Dual knife (KD620QR; Olympus Medical Systems, Tokyo, Japan) and IT knife nano (KD-612; Olympus Medical Systems, Tokyo, Japan) were used interchangeably according to circumstances in all cases. We used a coagrasper (FD-411QR; Olympus Medical Systems, Tokyo, Japan) for arterial bleeding that was otherwise unmanageable with the dissection knife. Sodium hyaluronate solution mixed with indigo carmine and adrenaline was used as injectate to lift the lesion. Carbon dioxide was used for insufflation. We used an ERBE electrosurgical unit, VIO300D (Erbe, Tübingen, Germany). The settings used were: Endo Cut mode I (effect 2, duration 2, interval 2) for mucosal incision and forced coagulation mode (effect 2, 45 W) for submucosal dissection and vessel coagulation.

-Fig. 1 shows a sub-circumferential laterally spreading tumor (LST) in the sigmoid colon that measures $170 \mathrm{~mm}$. Initially, the patient position was adjusted so that the tumor was on the opposite side of gravity, improving access to the submucosal layer after initial incision. The sodium hyaluronate solution was injected on the anal side of the tumor ( $\mathbf{F i g . 2}$ ). Using a dual knife, a horizontal mucosal incision on the anal side of the tumor was created as an entrance to create a straight tunnel towards the oral side, being careful not to excessively expand the

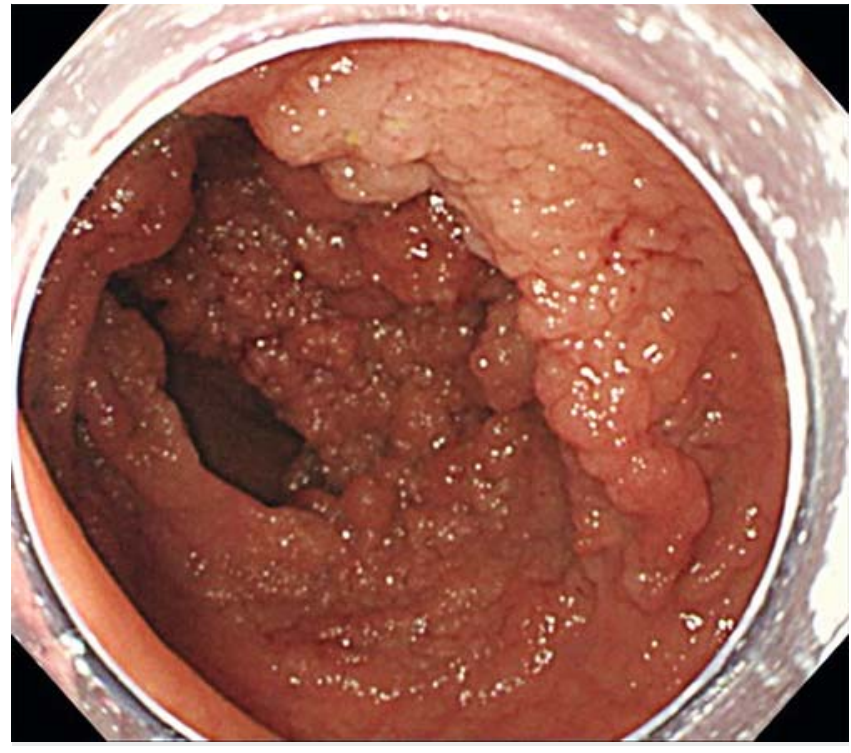

- Fig. 1 Laterally spreading tumor (LST) of the sigmoid colon measuring $170 \mathrm{~mm}$, with only a small area of spared normal mucosa.

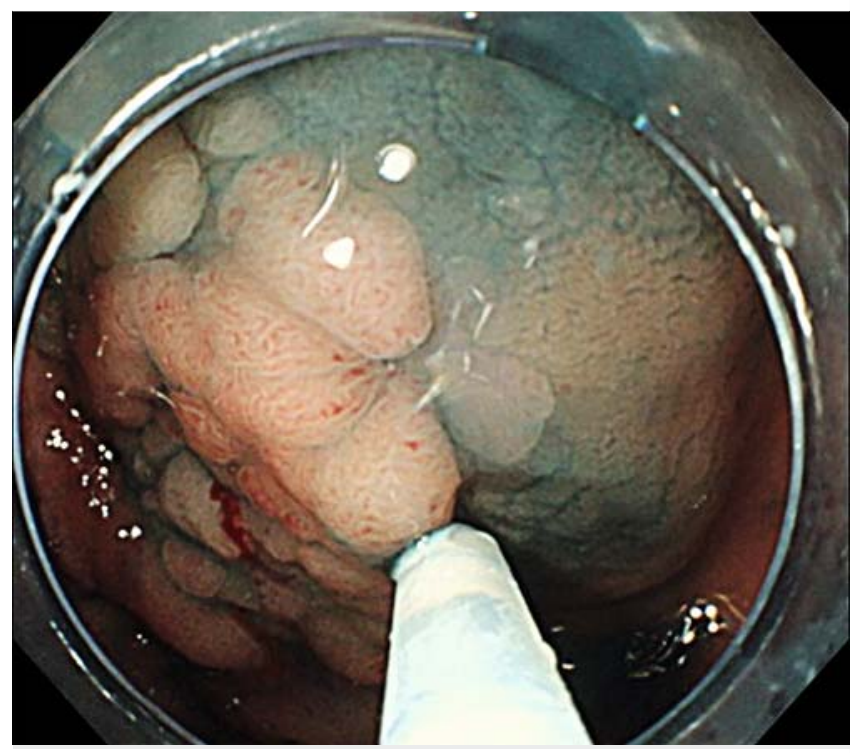

- Fig. 2 Local injection of hyaluronic acid mixed with indigo-carmine into the anal end of the lesion.

tunnel diameter horizontally, as that could compromise scope stability. Leaving a thin sheet of submucosal tissue in between, another straight tunnel was made adjacent to the first tunnel. The above procedure was repeated until the entire width of the tumor was undermined by the tunnels, leaving multiple sheets of submucosal tissues in between the tunnels, resembling a palisade ( $>$ Fig.3). Circumferential resection around the lesion was made after successful creation of the tunnels, after which the sheets of submucosal tissues were dissected with the dual knife, ultimately removing the tumor ( $\mathbf{F i g . 4}$ and $>$ Fig. 5). The specimen was then sent for pathological analysis by a pathologists unaware of the resection technique used. 


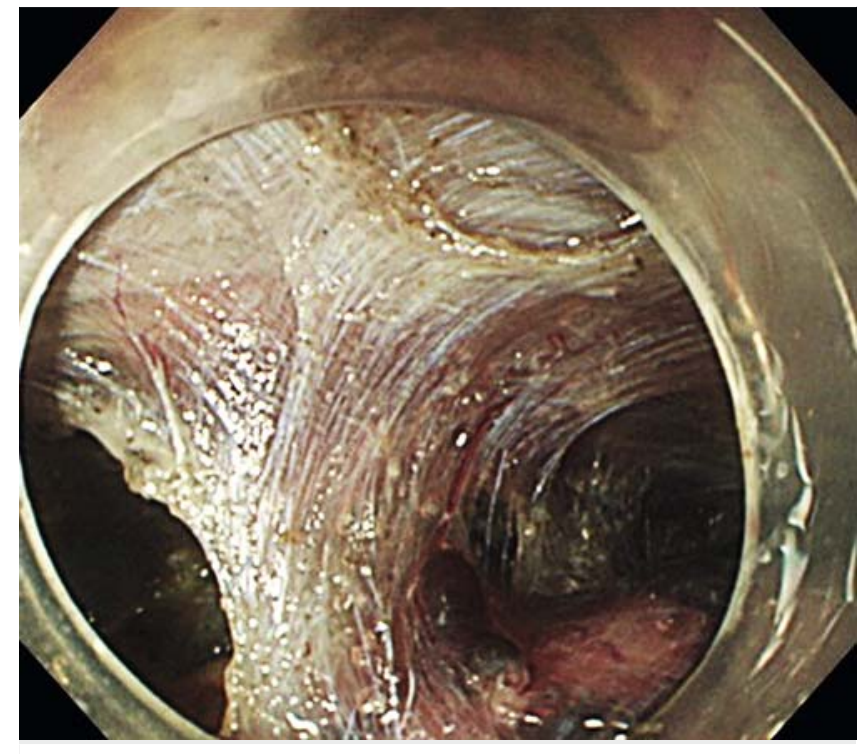

- Fig. 3 A sheet of submucosal tissue is left in between the two submucosal tunnels. These sheets of submucosa keep the tumor from collapsing onto the gravity-side of the lumen.

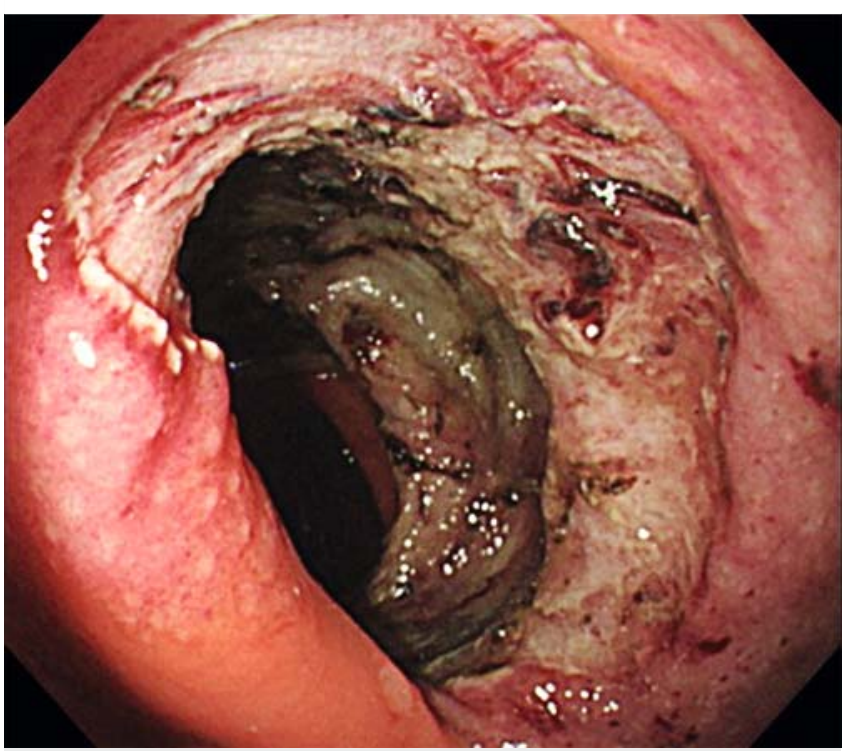

- Fig. 4 The whole lesion is resected, leaving a large mucosal defect.

\section{Outcomes}

The primary outcome of this study was the R0 resection rate. Secondary outcomes included en-bloc resection rate, rate of AEs, median submucosal dissection speed (SDS), submucosal dissection time (SDT), and local recurrence rate. SDT was calculated by dividing the lesion area by dissection time. We classified lesion size as follows. Full circumferential lesions extended over the full circumference of the colorectal lumen with no intervening normal mucosa in between the lesion edge, semi-circumferential lesions occupied over half of the lumen, and subcircumferential lesions we defined as in between these cases. The lesion area was calculated by multiplying half the length

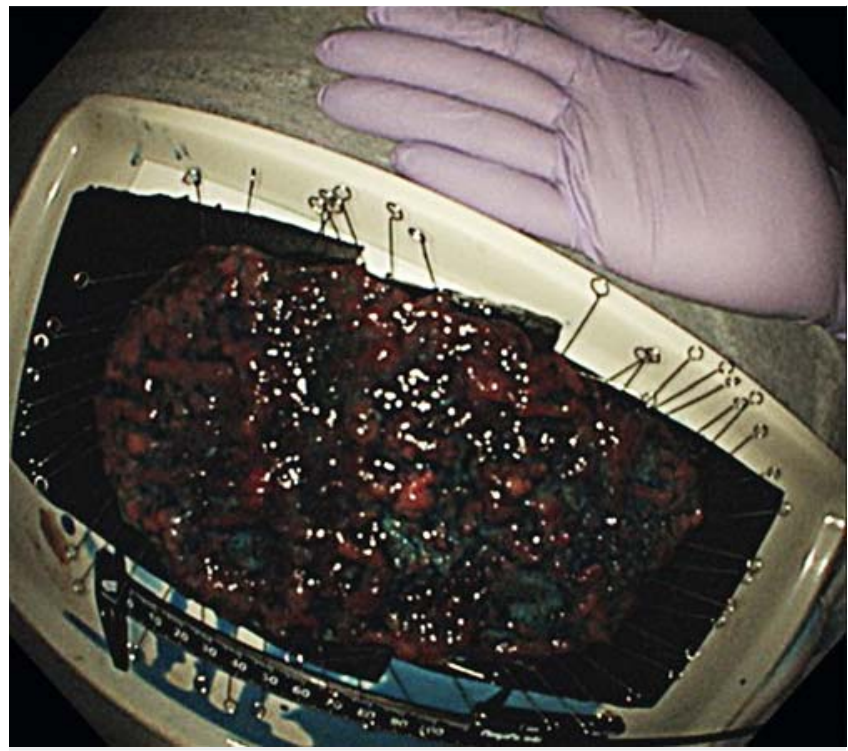

- Fig. 5 The dissected tumor pinned on rubber for pathological analysis

by half the width of the lesion and then multiplying the product by 3.14. The longest axis of the lesion was considered the length, and the axis perpendicular to the length was measured as the width. All procedures were performed by expert endoscopists, defined as operators who performed more than 80 colorectal ESD cases [5]. Five experts participated in the study.

A resected specimen was pinned and placed in $20 \%$ formalin. All specimens were sectioned serially in 2- to $3-\mathrm{mm}$ intervals and sent for histological evaluation and reported using the Vienna classification. R0 resection was defined as complete resection with negative lateral and vertical margins. En bloc resection was defined as one-piece resection of the tumor with free margins on macroscopic assessment. Immunohistopathological staining was performed to assess the presence of lymphovascular invasion. AEs assessed include delayed bleeding, perforation, and post-colorectal ESD coagulation syndrome (PECS). This was defined as local abdominal pain in the region corresponding to the site of the ESD within 4 days after ESD. We defined delayed bleeding as presence of marked bloody stool after treatment requiring hemostasis. Intraoperative perforation was defined as a full-thickness defect of the colorectal wall that was recognized by the endoscopist as a state in which connective tissue, adipose tissue, and/or serosa are visualized through the defect during ESD. Delayed perforation was defined as perforation of the colon that occurre after the scope had been withdrawn following the completion of ESD in which intraprocedural perforation did not occur. We defined post-ESD stricture as stenosis in which an endoscope failed to advance.

\section{Results}

A total of 11 patients were included in the study and of them, seven were men (64\%). > Table 1 lists the characteristics of the patients included in this study. Their median was 69 years 
- Table 1 Patient demographics.

\begin{tabular}{|l|l|c|}
\hline \multirow{2}{*}{ Age } & Median & $\mathbf{6 9}$ (43-86) \\
\hline \multirow{2}{*}{ Gender } & Male & 7 \\
\hline \multirow{2}{*}{$\begin{array}{l}\text { Number of submucosal } \\
\text { sheets }\end{array}$} & Female & 4 \\
\hline \multirow{2}{*}{ Location } & Fouree & 9 \\
\hline \multirow{2}{*}{ Morphology } & Five & 1 \\
\hline \multirow{2}{*}{ Circumferentiality } & Rectum & 1 \\
\hline & Colon & 4 \\
\hline \multirow{2}{*}{} & Pedunculated & 1 \\
\hline & Non-pedunculated & 10 \\
\hline & Full circumferential & 0 \\
\hline & Subcircumferential & 6 \\
\hline & Semicircumferential & 5 \\
\hline
\end{tabular}

(range 43 to 86). Seven lesions were located in the colon and four lesions in the rectum. The number of sheets made for dissection was three in nine cases, five in one case, and four in one case. Of the lesions, $91 \%$ were non-pedunculated in morphology. Six lesions were sub-circumferential, and five were semi-circumferential. None of the tumors were fully circumferential. We did not have any lesions with extensive submucosal fibrosis. - Table 2 shows outcomes of this study. The R0 resection rate was $45.6 \%$. The median SDT and SDS were 170 minutes, and $23.1 \mathrm{~mm}^{2} / \mathrm{min}$, respectively. The en bloc resection rate was $100 \%$. We had three acute AEs, including one patient with delayed bleeding with decline in hemoglobin of $1.8 \mathrm{~g} / \mathrm{dL}$, which was endoscopically managed without requiring transfusion, and two patients with PECS, both managed conservatively. No perforations occurred. We recorded one case of post-ESD stricture. Pathological evaluation demonstrated one adenoma with low-grade dysplasia, two with high-grade dysplasia, adenocarcinoma-in-adenomas, and two early cancers. There was one patient with venous invasion, but none of the patients had lymphatic invasion. The patient with adenocarcinoma-in-adenoma had a 6400- $\mu$ m submucosal invasion and declined surgery. He is undergoing surveillance with no signs of recurrence. The other patient had submucosal invasion of $1100 \mu \mathrm{m}$ and underwent surgical resection with no evidence of lymph node metastasis. This patient is also being carefully followed up with no recurrence. All 11 patients received follow-up colonoscopy, and all of the patients remain free of recurrence after a median observation period of 31 months.

\section{Discussion}

In our study, 11 large colorectal tumors growing over half the luminal circumference of the colon were successfully dissected with ESD using the palisade technique.
- Table 2 Outcomes of the palisade technique in dissecting large colorectal tumors.

\begin{tabular}{|c|c|}
\hline Outcome & \\
\hline Median lesion length (range), mm & $103(56-170)$ \\
\hline Median lesion area (range), mm² & $4219.4(2121.9-12677.8)$ \\
\hline Median SDT (range), min & $170(43-350)$ \\
\hline Median SDS (range), $\mathrm{mm}^{2} / \mathrm{min}$ & $23.1(10.2-77.5)$ \\
\hline R0 resection, n (\%) & $5(45.6 \%)$ \\
\hline En bloc resection, $\mathrm{n}(\%)$ & $11(100 \%)$ \\
\hline Perforation, n (\%) & $0(0 \%)$ \\
\hline Post ESD bleeding, n (\%) & $1(9.1 \%)$ \\
\hline PECS, n (\%) & $2(18.2 \%)$ \\
\hline Adverse events, n (\%) & $3(27.3 \%)$ \\
\hline \multicolumn{2}{|l|}{ Pathology } \\
\hline Adenoma, n (\%) & $3(27.2 \%)$ \\
\hline Adenocarcinoma in adenoma, $\mathrm{n}(\%)$ & $6(54.5 \%)$ \\
\hline Adenocarcinoma, n (\%) & $2(18.2 \%)$ \\
\hline \multicolumn{2}{|c|}{$\begin{array}{l}\text { SDT, submucosal dissection time; SDS, submucosal dissection speed; ESD, } \\
\text { endoscopic submucosal dissection; PECS, post-colorectal ESD coagulation } \\
\text { syndrome. }\end{array}$} \\
\hline
\end{tabular}

Treating large colorectal tumors is a challenge faced by endoscopists at many levels. We often recognize that despite large size, certain laterally spreading lesions are able to be curatively resected with endoscopic resection. If the endoscopist decides to treat the lesion endoscopically, a robust strategy is required, given the size and potential difficulty. One of the difficulties we face is the loss of traction during dissection of such large lesions. With a conventional ESD technique, the dissected lesion hangs into the lumen, which provides traction at the beginning of the dissection. However, the dissected lesion will eventually hang onto the contralateral wall, where the majority of the tumor weight gets supported. Such a reduction in the tumor weight weakens the gravitational pull on the submucosal tissue. Thus, traction is lost during dissection ( $\mathbf{F i g . 6 a ) . ~ T h e ~}$ palisade technique leaves sheets of submucosal tissues that anchor the lesion on the wall opposite to gravity, which prevents loss of traction on the submucosal tissue, allowing for more effective dissection ( $>$ Fig. 6 b).

While a high en bloc resection rate was observed, the R0 resection rate in our study was lower than reported for conventional colorectal ESD [6]. We feel this is due to the coagulation effect on the lateral margins, supported by the fact that we had no evidence of recurrence with endoscopic surveillance.

The size of the lesion is known to be an independent risk factor for adverse outcomes associated with colorectal ESD [1]. This may be due to the greater area of the submucosal layer exposed to thermal insults from the electrosurgical knives. This, together with the thin wall of the colon and rectum, results in an increased risk of perforation with ESD of these lesions. In 


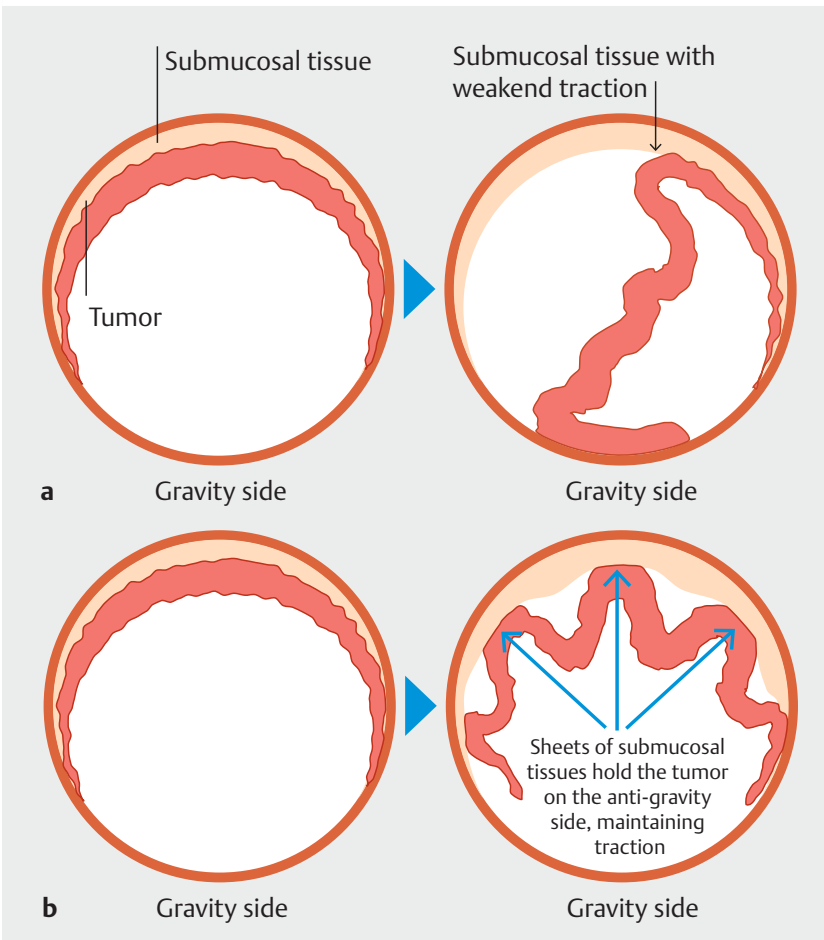

- Fig. 6 a Traction on submucosal tissue becomes weaker as the weight of the dissected tumor gets supported by the contralateral wall of the colon for the conventional method of ESD. This reduces gravitational pull leading to weakened traction on submucosal tissue. $\mathbf{b}$ In the palisade technique, the traction on submucosal tissue is maintained by preventing the tumor from hanging down onto the contralateral wall, allowing effective dissection.

that regard, our study demonstrated favorable results in comparison to conventional ESD, where the perforation rate is reported at $4.5 \%$ [7]. Rates of delayed bleeding, however, were higher than previously reported (2.4\% in Asian countries) [7, 8]. Half of the lesions in this study were located in the rectum and larger than $40 \mathrm{~mm}$, both of which are reported risk factors for post-ESD bleeding [9]. In all cases, the bleeding was successfully managed endoscopically.

PECS is thought to result from electrical current extending towards the serosa during ESD. This transmural burn may manifest as abdominal pain post-procedure. The occurrence of PECS has been reported to be $14.2 \%$ with independent risk factors including female gender, cecal lesions, and ESD procedure time $>90$ minutes [10]. The median procedure time in our study was 170 minutes, yet our series had two cases of suspected PECS, which were managed conservatively. One case was a rectal lesion in a female patient with a procedure time of 265 minutes, and the other case was a sigmoid lesion in a male patient with a procedure time of 350 minutes.

Previous studies report a high rate of stenosis in resection of lesions that grow over $90 \%$ of the luminal circumference, and $11.1 \%$ patients who were treated for rectal tumors that required resection of more than $90 \%$, but less than $100 \%$ of luminal circumference developed stricture after ESD [11]. Our study also had one case of post-ESD stenosis in a patient treated for a rectal lesion covering greater than $90 \%$ of the luminal circumference. The patient was preemptively treated with steroid enemas and also had endoscopic observation of the defect every 2 weeks to assess the healing process. He developed an asymptomatic stenosis after 1.5 months and had bougie dilation 10 times over 5 weeks.

Although we have demonstrated that the palisade technique is effective in treating large colorectal tumors, it may not be as effective in treating lesions located in the cecum, hepatic flexure, or in the splenic flexure, where a more perpendicular approach may be required, causing the lesion to tear. This can be avoided by creating sufficient submucosal space so that the scope tip can be inserted into the submucosal layer without exerting excessive force that could bluntly tear the specimen apart. Lesions such as non-granular laterally spreading tumors (LST-NG) may have more submucosal fibrosis. The palisade technique can be used to treat such lesions by creating tunnels on both sides of the fibrotic tissue so that the tissue is isolated, which makes the fibrosis more visible to the endoscopist, allowing for more precise dissection.

Limitations of the study include its retrospective nature and completion in only a single center. Without a control group, definitive conclusions cannot be drawn about the true effectiveness of this technique. Even though we compared our outcomes, such as $\mathrm{R} 0$ resection rate and rates of AEs with the historical control from previous studies, the results need to be interpreted carefully, as the background characteristics between the cohorts differ significantly. Our case numbers are low, as large lesions such as these are rare. There is a potential selection bias for selecting large tumors that are potential candidates for this technique. Furthermore, the fact that all of the ESDs are conducted by expert endoscopists may lead to overestimation of the effectiveness and feasibility of the technique. A prospective study comparing this technique to other methods of ESD for large lesions would be useful to assess its true effectiveness.

\section{Conclusion}

In conclusion, the palisade technique can be safe and effective for dissecting large colorectal tumors that would otherwise be difficult to treat with conventional ESD methods. Due to larger tumor size, delayed bleeding may occur at a higher rate than conventionally reported, but can be safely managed endoscopically. Further prospective studies for treatment of these large lesions is necessary.

\section{Competing interests}

The authors declare that they have no conflict of interest.

\section{References}

[1] Saito Y, Uraoka T, Yamaguchi Y et al. A prospective, multicenter study of 1111 colorectal endoscopic submucosal dissections (with video). Gastrointest Endosc 2010; 72: 1217-1225 
[2] Aslan F, Akpınar Z, Kucuk M et al. Multiple tunneling technique for treatment of rectal circumferential laterally spreading tumor with endoscopic submucosal dissection. VideoGIE 2017; 2: 32-34

[3] Watanabe T, Muro K, Ajioka Y et al. Japanese Society for Cancer of the Colon and Rectum (JSCCR) guidelines 2016 for the treatment of colorectal cancer. Int J Clin Oncol 2018; 23: 1-34

[4] Tanaka S, Kashida H, Saito Y et al. Guideline JGES guidelines for colorectal endoscopic submucosal dissection/endoscopic mucosal resection. 2015: https://pdfs.semanticscholar.org/e1a1/052c870a298fe0fe1b1e3a5b97e85d4a1cfc.pdf

[5] Pimentel-Nunes P, Dinis-Ribeiro M, Ponchon T et al. Endoscopic submucosal dissection: European Society of Gastrointestinal Endoscopy (ESGE) Guideline. Endoscopy 2015; 47: 829-854

[6] Fuccio L, Hassan C, Ponchon T et al. Clinical outcomes after endoscopic submucosal dissection for colorectal neoplasia: a systematic review and meta-analysis. Gastrointest Endosc 2017; 86: 74-86.e17
[7] Fuccio L, Ponchon T. Colorectal endoscopic submucosal dissection (ESD). Best Pract Res Clin Gastroenterol 2017; 31: 473-480

[8] Ohata K, Nonaka K, Minato Y et al. Endoscopic submucosal dissection for large colorectal tumor in a Japanese general hospital. J Oncol 2013: doi:10.1155/2013/218670 Available from: http://www.ncbi. nlm.nih.gov/pubmed/24072998

[9] Chiba H, Ohata K, Tachikawa J et al. delayed bleeding after colorectal endoscopic submucosal dissection: when is emergency colonoscopy needed? Dig Dis Sci 2019; 64: 880-887

[10] Arimoto J, Higurashi T, Kato $S$ et al. Risk factors for post-colorectal endoscopic submucosal dissection (ESD) coagulation syndrome: a multicenter, prospective, observational study. Endosc Int Open 2018; 6: E342-E349

[11] Hayashi T, Kudo S, Miyachi H et al. Management and risk factor of stenosis after endoscopic submucosal dissection for colorectal neoplasms. Gastrointest Endosc 2017; 86: 358-369 